\title{
Staatliche Schuldvermutung statt verfassungsrechtlicher Unschuldsvermutung. Wer überwacht die Wächter?*
}

Uwe Diercks," Polizeipräsidium Bonn

\section{Einleitung}

Seitdem der Bundesgesetzgeber in seiner Gesetzessprache in den $\$ \mathbb{S} 98 \mathrm{a}, 100 \mathrm{i}, 111$, 153 e, 163 d, 163 e, 163 f StPO, $\$ 4$ BKAGesetz sowie $\mathbb{} 31$ a BtM-Gesetz den hier verfassungsrechtlich anstößigen Begriff „Täter“ verwendet, ist es zu einem begriffsjuristischen Dammbruch gekommen. ${ }^{1}$

Nunmehr wurde diese den Rechtsstatus des Tatverdächtigen bzw. Beschuldigten missachtende Ausdrucksweise auch in Nordrhein-Westfalen bei der Polizeilichen Kriminalstatistik etabliert.

\section{Eingabe an den Petitionsausschuss des Landtages von Nordrhein- Westfalen}

Wegen dieses verfassungsrechtlich anstößigen Begriffs „Täter“ in der Polizeilichen Kriminalstatistik wandte sich der Verfas$\operatorname{ser}^{2}$ wie folgt an die Vorsitzende des Petitionsausschusses des Landtages von Nordrhein-Westfalen I. Howe:

„Im Interesse der Rechts- und Polizeikultur möchte ich von dem in Artikel 17 GG verbrieften Eingaberecht Gebrauch machen, um auf meines Erachtens bestehende Mängel in der Erfassung der Polizeilichen Kriminalstatistik (PKS) des Landes NordrheinWestfalen aufmerksam zu machen, die in der Praxis zu einem rechtlich unhaltbaren Zustand führen. Dem liegt folgender Sachverhalt zugrunde:

Als betroffener Polizeibeamter, der bei der polizeilichen Vorgangsbearbeitung seit Anfang Januar 2008 die neue PKS-Erfassung vornehmen soll, mache ich mir Sorgen, wie diese mit den Vorgaben der unabänderlichen verfassungsrechtlichen Prinzipien in Einklang zu bringen ist. Die Polizei des Landes Nordrhein-Westfalen muss hierbei die entsprechenden Richtlinien umsetzen, so dass die Zuständigkeit des Innenministers, Herrn Dr. Ingo Wolf, als oberster Dienstherr gegeben ist.

Zur Begründung möchte ich Folgendes ausführen:

Neben den handwerklichen Unzulänglichkeiten - die statistische Erfassung in zwei verschiedenen Intranet-Eingabeformularen und spätere Kontrolle durch Vorgesetzte dauert bei Delikten der Massenkriminalität oft länger als die Sachbearbeitung zur Kriminalitätsbekämpfung - ist befremdlich, dass die Personaldaten der ermittelten Tatverdächtigen / Beschuldigten in die Eingabemasken mit den vorgegebenen Begriffen ,Täter', ,Mittäter' bzw. ,Wiederholungstäter' einzufügen sind, wobei die gleichzeitige Verwendung des Wortes, Täter' mit den ,Beschuldigten / Tatverdächtigen' besonders irreführend ist: (...).

Gegen diese PKS-Erfassung bestehen erhebliche verfassungsrechtliche Bedenken ${ }^{3}$ :

Im Ermittlungsverfahren gibt es keinen, Täter`. Die Feststellung der Täterschaft obliegt allein dem Gericht im Hauptverfahren. Die Unschuldsvermutung verlangt, dass nur im prozessordnungsgemäßen Verfahren und erst, nachdem ein gerichtlicher Nachweis der Schuld erbracht ist, angenommen werden kann, dass ein Tatverdächtiger auch der Täter ist. Darin ist weit mehr als eine bloße Sprachregelung zu sehen.

Das Strafprozessrecht regelt den Gang der Klärung eines Verdachts und ist geprägt von herkömmlichen rechtlichen Standards, die insbesondere im polizeilichen Umgang mit Tatverdächtigen und Beschuldigten zu respektieren sind. Für noch nicht rechtskräftig abgeschlossene Strafverfahren gilt das Prinzip der Unschuldsvermutung, das eine nähere Ausgestaltung in Art. 6 Abs. 2 der Europäischen Konvention zum Schutze der Menschenrechte und Grundfreiheiten (EMRK) erfahren hat. Diese Bestimmung gilt - nach Ratifizierung durch den Bundesgesetzgeber - in Deutschland als unmittelbar geltendes innerstaatliches Recht. Danach wird vermutet, dass der wegen einer strafbaren Handlung Beschuldigte bis zum gesetzlichen Nachweis seiner Schuld unschuldig ist.

Es besteht Übereinstimmung, dass dem heute weltweit in allen Rechtsstaaten anerkannten Prinzip der Unschuldsvermutung verfassungsrechtliche Bedeutung zukommt. Die Unschuldsvermutung - die darauf abzielt, den Verdächtigen vor diskriminierender und willkürlicher Behandlung zu schützen - ist
Bestandteil der verfassungsrechtlichen Prinzipien der Unantastbarkeit der Menschenwürde (Art. 1 Abs. 1 GG) und der Rechtsstaatlichkeit (Art. 20 Abs. 3 und Art. 28 Abs. 1 Satz 1 GG). Durch ihre Einbeziehung in den Bürgerrechtspakt gehört sie auch zu den allgemeinen Regeln des Völkerrechts (Art. 25 GG).

Somit kommt der Unschuldsvermutung Verfassungsrang zu. Darüber hinaus gehört sie zu den ,unabänderlichen' Rechtsprinzipien, die den ,Kern der Verfassung' ausmachen und die Art. 79 Abs. 3 GG der verfassungsgesetzgebenden Gewalt entzogen hat.

Diese Maxime eines rechtsstaatlichen Strafverfahrens gilt vom Beginn der Ermittlungen an bzw. mit Entstehen des Tatverdachts bis zum rechtskräftigen Verfahrensabschluss, in welchem sie entweder widerlegt oder bestätigt wird.

Während des gesamten Verfahrens ist die Unschuldsvermutung eine konstante Größe. Der Schutz, den sie gewährt, gilt generell; eine Relativierung auf Grund besonderer Umstände des Falles ist ausgeschlossen. Denn bis zum Eintritt der Rechtskraft ist kein normativ hinreichendes Maß an Sicherheit darüber erreicht, wie die Sach-, Rechtsund Beweislage endgültig einzuschätzen ist.

Da die Verhinderung außerjustizieller Schuldzuschreibungen $\mathrm{zu}$ den originären Aufgaben der Unschuldsvermutung gehört, darf niemand, kein noch so Verdächtiger außerhalb des prozessordnungsgemäßen Verfahrens oder vor seinem rechtskräftigen Abschluss als Straftäter bezeichnet werden. Erst die rechtskräftige Verurteilung stellt eine feste Beziehung zwischen Tat und Täter her; denn vor der Verurteilung besteht hinsichtlich der Straftat ein Zustand schwebender Unwirksamkeit. Der klare Trennungsstrich zwischen dem Rechtstreuen und dem Rechtsbrecher wird erst mit einem rechtskräftigen Strafurteil gezogen. Somit ist die Widerlegung der Unschuldsvermutung allein dem erkennenden Strafgericht vorbehalten und darf nicht im Vorfeld der gerichtlichen Entscheidung von anderen beteiligten Organen vorweggenommen werden. 
Als Adressaten soll die Unschuldsvermutung alle staatlichen Organe - wie Staatsanwaltschaft und Polizei, aber auch Gericht und insbesondere Gesetzgeber - als Verfassungssatz binden.

Vor der rechtskräftigen Verurteilung gebietet die Unschuldsvermutung größtmögliche Zurückhaltung und gesteigerte Sorgfalt bei allen Eingriffen in die Rechtssphäre des Verdächtigen bzw. Beschuldigten.

Niemand ist berechtigt - auch PKS-Erfasser der Polizei nicht -, einen (lediglich) Tatverdächtigen als (bereits überführten und schuldig gesprochenen Straf-) ,Täter ‘ zu bezeichnen. Denn die Unschuldsvermutung hat die Aufgabe, staatliche Eingriffe in die Rechtssphäre des (bloß) Tatverdächtigen zu begrenzen und ihn gegenüber übermächtiger Staatsgewalt zu schützen.

Insbesondere beim juristisch nicht vorgebildeten Staatsbürger wird der Eindruck vermittelt, dass es sich bei dem als, Täter` bezeichneten bzw. gekennzeichneten Menschen um den überführten und verurteilten Straftäter handelt, so dass der Tatverdächtige (vor-)verurteilt, sozial angeprangert und stigmatisiert wird. Damit wird die sozialethisch deklassierende Wirkung der Bestrafung vorweggenommen und die Würde des Tatverdächtigen - über die durch das Schuldfeststellungsverfahren ohnehin bedingten Persönlichkeitseingriffe hinaus - angetastet.

Wegen dieser Suggestivwirkung in noch nicht rechtskräftig abgeschlossenen Strafverfahren darf die Gefahr der psychologischen Beeinflussung der Allgemeinheit und der am Verfahren bzw. an der Rechtsfindung Beteiligten nicht unterschätzt werden.

Die Verwendung der Begriffe ,Täter', ,Mittäter' bzw. ,Wiederholungstäter' bei der PKS-Erfassung im Ermittlungsverfahren hat die bedenkliche Folge, dass die Ermittlungsorgane unter bestimmten Voraussetzungen durch die entsprechenden Richtlinien gewissermaßen dazu amtlich aufgefordert werden, verdächtige bzw. beschuldigte Personen bereits als ,Täter' anzusehen.

Der leichtfertige Umgang der Sprache in den Richtlinien der PKS-Erfassung - wo Sensibilität, rechtsstaatliche Präzision, Perfektion und eine sachgerechte, jeden Anschein einer unzulässigen Schuldzuweisung vermeidende Polizeiliche Kriminalstatistik-Sprache erwartet werden kann - lässt auf mangelndes Problembewusstsein hinsichtlich der Verwendung des verfassungsrechtlich anstößigen Begriffs ,Täter‘ im Ermittlungsverfahren schließen. Denn mit der in Art. 6 Abs. 2 EMRK spezialgesetzlich normierten Unschuldsvermutung als allgemeine Rechtsvermutung - die nur durch eine rechtskräftige strafgerichtliche Verurteilung widerlegt werden kann, im Übrigen aber nicht auf den Strafprozess beschränkt ist, sondern interdisziplinär ernst genommen für das gesamte Rechtsleben Gültigkeit beansprucht - verbindet sich wie mit kaum einer anderen Maxime unseres Verfahrensrechts der Gedanke besonderer rechtsstaatlicher Fairness.

Im Hinblick auf die Reichweite der interdisziplinär ernst genommenen Unschuldsvermutung sind die Ausführungen des Bundesverfassungsgerichts ${ }^{4}$ von besonderer Bedeutung:

,Dem Täter müssen deshalb Tat und Schuld nachgewiesen werden. Bis zum gesetzlichen Nachweis der Schuld wird seine Unschuld vermutet.

In der Phase des Ermittlungsverfahrens (!) kann somit eine entsprechende Person lediglich Tatverdächtiger bzw. Beschuldigter sein, nicht jedoch ,Täter‘.

Aufgabe der Exekutive ist es, die Gesetze anzuwenden. Hierbei hat die Polizei die Aufgabe, Straftaten zu erforschen $(\mathbb{\$} 163$ Abs.1 StPO) und Ermittlungen gegen Tatverdächtige durchzuführen.

In unserer freiheitlichen demokratischen Grundordnung repräsentiert die Polizei einen Teil der Staatsgewalt und ist somit Garant für die Innere Sicherheit. In einer Gesellschaft, in der der Mensch im Mittelpunkt steht und die Unantastbarkeit der Menschenwürde oberster Verfassungsgrundsatz ist, wird der Staat gerade auch an seiner unvoreingenommenen, unparteiischen und weder präjudizierenden noch moralisierenden Polizei gemessen. Deshalb ist es geradezu verpflichtend, dass die verfassungsrechtlichen Prinzipien sowohl im polizeilichen Umgang mit Tatverdächtigen bzw. Beschuldigten als auch bei der PKS-Erfassung eingehalten werden.

Einerseits verlangt die verfassungsmäßige Ordnung von jeder Polizeibeamtin bzw. jedem Polizeibeamten Loyalität gegenüber dem Staat mit seinen Gesetzen und Richtlinien. Andererseits binden die unverfügbaren verfassungsrechtlichen Prinzipien der Unantastbarkeit der Menschenwürde und der Rechtsstaatlichkeit sowie die allgemeinen Regeln des Völkerrechts alle Angehörigen der Polizei bei der Ausübung ihres Berufs, so dass auf Grund der Richtlinien zur PKSErfassung Gesetzesanwender und PKS-Erfasser in eine Konfliktsituation gebracht werden könnten. Unter Zugrundelegung des straftatsystematischen Sprachgebrauchs - der Verfassungsprinzipien und sonstige Rechtsgrundsätze, wie beispielsweise das Rechtsstaatsprinzip, das Schuldprinzip, den Grundsatz der Unschuldsvermutung in die strafrechtliche Praxis umsetzt - dürften Rechtsanwender und PKS-Erfasser in der Phase des Ermittlungsverfahrens (!) lediglich die Begriffe ,Verdächtiger' bzw. ,Beschuldigter' benutzen, während die derzeitige PKS-Erfassungs-Sprache auch die in diesem Verfahrensstadium verfassungsrechtlich anstößigen Begriffe ,Täter', ,Mittäter` und ,Wiederholungstäter' verwendet. Diese Verfahrensweise ist eines Rechtsstaates unwürdig.

Folglich ist eine begriffliche Klarstellung für Grundrechts- und Hoheitsträger dringend erforderlich. Unter Berücksichtigung der rechtstheoretischen und praxisbezogenen Aspekte ist die Verwendung des Betriffs ,Täter' bei der PKS-Erfassung im Ermittlungsverfahren weder mit dem Grundsatz der Unschuldsvermutung noch mit der Erwartungshaltung der Bürger und Beamten von der ,Polizei als Säule des Rechtsstaates ${ }^{6}$ in Einklang zu bringen. Deshalb sollte der Sprachgebrauch in den entsprechenden Richtlinien auch im Interesse der europäischen Rechtskultur konventions-, verfassungs- und grundrechtskonform geändert werden.

Als Vorsitzende des Petitionsausschusses und ,Anwältin des Bürgers' sind Sie, sehr geehrte Frau Howe, in unserer parlamentarischen Demokratie dazu berufen, Unrecht zu unterbinden. Sollten Sie nach Prüfung der Sach- und Rechtslage zu der Auffassung gelangen, dass mein Anliegen begründet und Abhilfe notwendig ist, bitte ich Sie, der Landesregierung eine entsprechende Initiative zu empfehlen.“

\section{Rechtsauffassung des Petitionsausschusses des Landtages von Nordrhein-Westfalen}

Die Präsidentin des Landtages von Nordrhein-Westfalen ${ }^{5}$ teilte - unter Einbeziehung der Stellungnahme des Innenministeriums ${ }^{6}$ - den vom Petitionsausschuss in seiner Sitzung vom 15.4.2008 gefassten Beschluss - der identisch mit dem IM-Beschlussvorschlag ist - mit: 
„Der Maskenname ,Täter' und die Datenfeldbezeichnung ,Täter alleinhandelnd“ werden im Integrationsverfahren-Polizei (IGVP) / Polizei-Vorgangs-Programm (PVP) in ,Tatverdächtiger' bzw. ,Tatverdächtiger alleinhandelnd' geändert. Das Datenfeld ,Internationaler Straftäter' wird aus beiden Anwendungen ersatzlos entfernt. Hinsichtlich der Katalogbegriffe, Wiederholungstäter', ,Mittäter', ,Anstifter ' und ,Gehilfe' werden Formulierungen erarbeitet, die den Verdachtsstatus der Zuschreibungen im IGVP/PVP deutlich herausstellen.

In der polizeilichen Kriminalstatistik werden die Begriffe ,Täter‘, ,Mittäter` bzw. ,Einzeltäter' zur Erläuterung kriminologischer oder strafrechtlicher Definitionen, zur Erklärung von Grenzfällen und zur Verdeutlichung der kriminalstatistischen Zuordnung von Sachverhalten genutzt. Sie dienen, ohne jeden Bezug zu konkreten Personen, lediglich zur Klarstellung von Tatbeständen. Ein Änderungsbedarf ist daher nicht erkennbar.

Der Petitionsausschuss sieht somit keine Veranlassung, der Landesregierung (Innenministerium) darüber hinaus Maßnahmen zu empfehlen.“

\section{Einwände}

Hinsichtlich der Stellungnahmen des Innenministeriums des Landes Nordrhein-Westfalen und des Bonner Polizeipräsidenten ${ }^{7}$ sowie des mitgeteilten Beschlusses des Petitionsausschusses gab der Verfasser der Präsidentin des Landtages von Nordrhein-Westfalen R. van Dinther folgende Einwände ${ }^{8} \mathrm{zu}$ bedenken:

\section{„1. Beamtenrechtliche Vorgaben}

Mein in dieser Angelegenheit ebenfalls am 14.1.2008 auf dem Dienstweg eingereichtes Schreiben an den Innenminister des Landes Nordrhein-Westfalen Dr. I. Wolf wurde am 30.1.2008 von der Leitenden Kriminaldirektorin G. Hewer-Brösch mit der befremdlichen Rechtsauffassung - dass ich dazu nicht berechtigt sei - zurückgewiesen, was auf Grund der bestehenden Gesetzeslage ( 179 Landesbeamtengesetz NordrheinWestfalen) nicht nachvollziehbar ist. Trotz des Hinweises auf das LBG NRW, meiner erneut geäußerten Bitte (Anlage), der übergeordneten Bedeutung und der Eilbedürftigkeit wurde die Weiterleitung dieses Schreibens an den zuständigen obersten Dienstherrn der Polizei vom Bonner Polizeipräsidenten W. Albers für nicht erforderlich erachtet (Anlage). Dieses Schreiben verblieb bis heute beim Polizeipräsidenten Bonn.

Anmerken möchte ich im übrigen, dass ich die mehrfach vom Innenministerium - insbesondere vom Leitenden Ministerialrat Wesseler - vorgenommene falsche Schreibweise des Namens des Petenten als Missachtung der Person deute (Anlage).

\section{Verwendung des Begriffs ,Täter}

Der Auffassung, dass im Bereich der Polizeilichen Kriminalstatistik (PKS) kein (weiterer) Änderungsbedarf erkennbar sei, kann unter Berücksichtigung der rechtstheoretischen und praxisbezogenen Aspekte ${ }^{9}$ meines Erachtens nicht gefolgt werden: Bei der strafprozessrechts-dogmatischen Diskussion um den ,Täter'-Begriff fällt auf, dass die Lehre ${ }^{10}$ davon ausgeht, dass entsprechende Personen - in der Phase des polizeilichen Ermittlungsverfahrens (!) - lediglich Tatverdächtige bzw. Beschuldigte sein können, nicht jedoch ,Täter'. Auch im Bereich der Polizeilichen Kriminalstatistik gibt es keinen rechts-(staats-)freien Raum. Dies möchte ich nachfolgend verdeutlichen:

\section{Kluft zwischen Theorie und Praxis}

Bei der PKS-Erfassung besteht eine verfassungsrechtlich fragwürdige Kluft zwischen Theorie (LKA-Richtlinien ${ }^{11}$, IM-Runderlass ${ }^{12}$ ) und Praxis (LZPD-Eingabehinwei$\left.\mathrm{se}^{13}\right)$ :

Einerseits wird - bereits seit 25 Jahren - hervorgehoben, dass die PKS eine Zusammenfassung aller der Polizei bekannt gewordenen strafrechtlichen Sachverhalte unter Beschränkung auf ihre erfassbaren wesentlichen Inhalte sei, wobei sie im Interesse einer wirksamen Kriminalitätsbekämpfung $\mathrm{zu}$ einem überschaubaren und möglichst verzerrungsfreien Bild der angezeigten Kriminalität führen solle. ${ }^{14} \mathrm{Im}$ Einzelnen diene die PKS der

- Beobachtung der Kriminalität und einzelner Deliktsarten, des Umfangs und der Zusammensetzung des Tatverdächtigenkreises sowie der Veränderung von Kriminalitätsquotienten,

- Erlangung von Erkenntnissen für vorbeugende und verfolgende Verbrechensbekämpfung, organisatorische Planungen und Entscheidungen sowie kriminologisch-soziologische Forschungen und kriminalpolitische Maßnahmen.

In Monatszeiträumen seien die von der Polizei bearbeiteten Verbrechen und Vergehen einschließlich der mit Strafe bedrohten Versuche gemäß Straftatenkatalog und die von der Polizei ermittelten Tatverdächtigen zu erfassen. Tatverdächtig sei dabei jeder, der nach dem polizeilichen Ermittlungsergebnis aufgrund zureichender tatsächlicher Anhaltspunkte verdächtig sei, eine rechtswidrige (Straf-) Tat begangen zu haben. Dazu seien auch ,Mittäter ${ }^{15}$ zu zählen.

Verfassungsrechtlich anstößig ist hier die Bezeichnung ,Mittäter'; denn wenn es im Stadium des fehleranfälligen Ermittlungsverfahrens ${ }^{16}$ noch keinen ,Täter' gibt, kann es bei der PKS-Erfassung erst recht auch keinen ,Mittäter' geben. Sachgerecht könnten hier die Begriffe Mittatverdächtiger bzw. Mitbeschuldigter ${ }^{17}$ sein.

Andererseits wird bei den - offensichtlich ,mit heißer Nadel gestrickten' - neuen PKS-Erfassungsvorgaben ${ }^{18}$ - mit dem Integrationsverfahren-Polizei (IGVP) und dem Polizei-Vorgangs-Programm (PVP) - darauf hingewiesen, dass durch den Bund eine grundsätzliche Neuschlüsselung der Delikte (sechsstellig) und eine neue Erfassungsweise in Form eines Einzeldatensatzes eingeführt worden sei. Durch die Polizei NRW erfolge die Ersterfassung der notwendigen Daten über das Modul PVP mit anschließendem Export in die Transferdatenbank. Die fachliche Qualitätssicherung sowie der Änderungsdienst seien über PKS-online 2 vorzunehmen.

In verfassungsrechtlich bedenklicher Weise wird sodann die Vorgehensweise zur Eingabe von ,Täterdaten ${ }^{19}$ beschrieben: ,Bei Aufruf der Folgemasken erhalten Sie wie gewohnt eine Auswahlmöglichkeit zur Übernahme bereits erfasster Personen oder zur Neuanlage von Personendaten. Die Eingabemaske, Täter erfassen / zuordnen ${ }^{20}$ (mit den vorgeschlagenen personenbezogenen Daten des Beschuldigten) wird erläutert: ,Neben den Standardfeldern zu Personen befinden sich auf der Maske zu den Täterpersonalien im unteren Bereich PKS-spezifische Felder (Wiederholungstäter, Intern. Straftäter, Mittäter).' Zum Begriff ,Täter ${ }^{\text {21 }}$ (-Eingabemaske mit Personalien des Beschuldigten) folgt die Erklärung: ,... das Feld ,Tatbeteiligung' steht in Abhängigkeit zu dem korrespondierenden Eintrag bei den Falldaten und der Anzahl der erfassten Täter. (...) Auf der Maske ,Täter' speichern Sie mit , $\mathrm{OK}^{\prime}$ die Daten und gelangen wieder in die Eingabemaske.' Sodann erfolgt die Bestätigung der Anzahl der ,Täter' (mit den personenbezogenen Daten). Es wird ferner darauf hingewiesen, dass nach Eingabe 
aller erforderlichen Daten mit einem ,OK“ in der Einstiegsmaske die automatische Generierung und Übertragung der Datensätze erfolge. $^{22}$

Erklärungsbedürftig und besonders irreführend erscheint - wegen der gleichzeitigen Verwendung der Begriffe, Täter' und Tatverdächtige - das vom Landeskriminalamt NRW aufgeführte theoretische Beispiel zu den Grenzen der natürlichen Handlungseinheit: ${ }^{23}$,Nach Sachbeschädigung an Kfz wird der Tatverdächtige vom Geschädigten verfolgt, der Täter bedroht und verletzt den Geschädigten.

Das Landeskriminalamt ${ }^{24}$ erläutert, dass es die Aufgabe habe, die statistischen Daten zu sammeln, sie in Zusammenarbeit mit dem Landesamt für Zentrale Polizeiliche Dienste NRW aufzuarbeiten und die erforderlichen Tabellen zu erstellen, wobei es durch entsprechende Kontrollmaßnahmen die Datenqualität gewährleiste. In Verbindung mit dem Begriff, echte Tatverdächtigenzählung ${ }^{25}$ wird erwähnt, dass - um den datenschutzrechtlichen Belangen zu genügen - für die PKS die Datenfelder (Geburts- und Vornamen, Geburtsdatum) bundeseinheitlich anonymisiert würden. Unverständlich erscheint jedoch, warum in der Praxis die ,echte Täterzählung‘ vorgenommen und weitergeleitet wird.

Mitgeteilt werden ferner die technischen Schnittstellen zu den Landeskriminalämtern anderer Bundesländer sowie die Anlieferung der Einzeldatensätze an das Bundeskriminalamt, das die Jahresstatistik der PKS der Bundesrepublik Deutschland zusammenfasse und sie Berechtigten zur Verfügung stelle sowie den Beitrag für die Internationale Kriminalpolizeiliche Statistik erstelle. ${ }^{26}$

Im März 2008 wurde die Kriminalstatistik 2007 für Nordrhein-Westfalen vom Innenminister ${ }^{27}$ und - exemplarisch für die Kreispolizeibehörde Bonn - vom Bonner Polizeipräsidenten ${ }^{28}$ sowie einer Leitenden Kriminaldirektorin der Öffentlichkeit vorgestellt: Die Presse berichtete (unwidersprochen) darüber, dass sich die Zahl der betrunkenen jugendlichen und heranwachsenden ,Gewalttäter ${ }^{629}$ mehr als verdoppelt habe und im Bereich der Straßenkriminalität 52,6 Prozent der ,Täter ${ }^{630}$ unter 21 Jahren alt gewesen seien.

Insbesondere herausragende Repräsentanten aus dem Bereich der Inneren Sicherheit - mit Vorbildfunktion für Bürger und Beamte - sollten - da sie sich auf Grund ihrer Stellung einen ,Amtsbonus ' verschaffen und folglich ihren Aussagen eine erhöhte Aufmerksamkeit sowie ein besonderes Gewicht zugemessen wird ${ }^{31}$ - die von der Polizei ermittelten Personen als Tatverdächtige bzw. Beschuldigte bezeichnen und nicht als ,Täter` kennzeichnen. Denn die Polizeiliche Kriminalstatistik ist nicht mit der Strafverfolgungsstatistik der Justiz ${ }^{32}$ vergleichbar, bei deren Vorstellung berechtigterweise von (überführten und schuldig gesprochenen Straf-) ,Tätern ${ }^{33}$ gesprochen werden kann.

Die alte Frage: ,Quis custodiet custodientes? - Wer überwacht die Wächter? ${ }^{34}$ ist heute berechtigter denn je.

\section{Gesetzliche Vorgaben}

Als Teil der öffentlichen Verwaltung ist die amtliche Statistik - die für die politische Willensbildung in der Gesellschaft die notwendigen Informationen liefert - an die gesetzlichen Regeln für das Verwaltungshandel gemäß Art. 20 Abs. 3 GG gebunden und hat somit Gesetz und Recht ${ }^{35}$ zu beachten.

Die amtliche Statistik findet ihre rechtliche Verankerung insbesondere im Bundesstatistikgesetz (BStatG). Für die amtliche Statistik gelten gemäß $\mathbb{1}$ BStatG - dieser Vorschrift kommt insoweit grundrechtssichernde Funktion $\mathrm{zu}^{36}$ - die Grundsätze der Neutralität, Objektivität und wissenschaftlichen Unabhängigkeit, wobei sie die Daten unter Verwendung wissenschaftlicher Erkenntnisse und unter Einsatz der jeweils sachgerechten Methoden und Informationstechniken gewinnt.

Vorliegend werden sowohl der sich hieraus - auf Bundesebene - ergebende Rechtsanspruch der Öffentlichkeit auf Wahrheit, Neutralität und Objektivität als auch die geforderte Daten-Gewinnung unter Verwendung wissenschaftlicher Erkenntnisse und Anwendung sachgerechten Methoden missachtet.

Zudem werden aber auch - auf Landesebene - die Vorgaben der PKS-Richtlinien (,wesentlicher Inhalt', ,verzerrungsfreies Bild‘, ,fachliche Qualitätssicherung', ,Datenqualität gewährleisten“, ,Tatverdächtigenkreis', ,Erkenntniserlangung für ... kriminalpolitische Maßnahmen') ${ }^{37}$ nicht sachgerecht erfüllt. Soweit Eingabehinweise und Bedienungsanleitung der PKS nicht die technischen Datenfeldbezeichnungen widerspiegeln, dürfte ein Verstoß gegen die Grundsätze der ordnungsgemäßen Datenverarbeitung nach $\ 10$ Abs. 2 Nr. 3 Datenschutzgesetz Nordrhein-Westfalen vorliegen. Für die Kontrolle der Einhaltung dieser
Vorschrift durch die Landesbehörden ist die Landesbeauftragte für Datenschutz und Informationsfreiheit zuständig.

Die polizeilich erhobenen personenbezogenen Daten der Tatverdächtigen bzw. Beschuldigten werden hier meines Erachtens im Rahmen der Polizeilichen Kriminalstatistik - beim Einarbeiten, Verwalten, Weiterleiten und bei der Vorstellung in der Öffentlichkeit - in unzulässiger Weise mit den in der Phase des Ermittlungsverfahrens verfassungsrechtlich anstößigen Begriffen ,Täter', ,Mittäter ${ }^{6}$ bzw. ,Wiederholungstäter' verknüpft.

Im strafrechtlichen Ermittlungsverfahren sind die strafprozessualen Grundsätze ${ }^{38}$ - insbesondere der Rechtsstatus ${ }^{39}$ des Tatverdächtigen bzw. Beschuldigten - zu beachten. Gerade in diesem sensiblen Bereich haben Bürger und Beamte einen Anspruch auf Wahrheit. Wahr ist jedoch, dass die hier erwähnten Personen in diesem konkreten Strafverfahren und in diesem Stadium des Ermittlungsverfahrens nicht (bzw. noch nicht) rechtskräftig verurteilt ${ }^{40}$ wurden und somit auch nicht als ,Täter' ausgewiesen werden dürfen. Der Öffentlichkeit wird ein falsches, verzerrtes Bild ${ }^{41}$ vermittelt, nämlich dass es der Polizei gelungen sei, alle bekannt gewordenen Straftaten bestimmten ,Tätern` zuzuordnen. Eigentlich steht zu diesem Zeitpunkt auch noch nicht fest, ob die behauptete Straftat tatsächlich auch eine Straftat ist. Das Gleiche gilt für das Opfer. Deshalb sollten Sensibilität, Wahrheit, Präzision und eine sachgerechte, jeden Anschein einer unzulässigen Schuldzuschreibung vermeidende (Amts-)Sprache ${ }^{42}$ bei den Richtlinien, der Erfassung und der Vorstellung (in der Öffentlichkeit) der PKS geradezu verfassungsrechtlich verpflichtend sein.

\section{Konventions-, verfassungs- und grundrechtliche Vorgaben}

Um einen Eingriff in die Rechtssphäre des Bürgers zu vermeiden, sind bei den Richtlinien, der Erfassung und der Vorstellung der PKS die konventions-, verfassungs- und grundrechtlichen Vorgaben zu beachten:

\subsection{Unschuldsvermutung, Menschenwürde und Rechtsstaatlichkeit}

Insbesondere die personenbezogene datenmäßige Verknüpfung von Tatverdächtigen bzw. Beschuldigten im Rahmen der PKS-Erfassung mit den - in der Phase des Ermittlungsverfahrens (!) - anstößigen Begriffen ,Täter', ,Mittäter‘ bzw. ,Wiederho- 
lungstäter' verletzt die mit Verfassungsrang ausgestattete Unschuldsvermutung (Art. 6 Abs. 2 EMRK) ${ }^{43}$ sowie die verfassungsrechtlichen Prinzipien der Unantastbarkeit der Menschenwürde (Art. 1 Abs. 1 GG) und der Rechtsstaatlichkeit (Art. 20 Abs. 3 und Art. 28 Abs. 1 GG); denn niemand ist berechtigt, einen (lediglich) Tatverdächtigen bzw. Beschuldigten als (bereits überführten und schuldig gesprochenen Straf-) ,Täter' zu bezeichnen bzw. zu kennzeichnen. ${ }^{44}$

\subsection{Allgemeines Persönlichkeitsrecht und Recht auf informationelle Selbstbestimmung}

Die dargestellte personenbezogene datenmäßige Verknüpfung von Tatverdächtigen bzw. Beschuldigten mit dem Begriff, Täter` verletzt ferner das zum allgemeinen Persönlichkeitsrecht (Art. 2 Abs. 1 GG in Verbindung mit Art. 1 Abs. 1 GG) zählende und verfassungsrechtlich gewährleistete Recht auf informationelle Selbstbestimmung ${ }^{45}$, das den Schutz des Einzelnen gegen unbegrenzte Erhebung, Speicherung, Verwendung und Weitergabe seiner persönlichen Daten umfasst.

Im Mittelpunkt der grundgesetzlichen Ordnung stehen Wert und Würde der Person, die in freier Selbstbestimmung als Mitglied einer freien Gesellschaft wirkt. ${ }^{46}$ Mit dem Recht auf informationelle Selbstbestimmung wären eine Gesellschaftsordnung und eine diese ermöglichende Rechtsordnung nicht vereinbar, in der Bürger nicht mehr wissen können, wer was wann und bei welcher Gelegenheit über sie weiß. ${ }^{47}$

Die PKS-Erfassung führt zu einer mit der Würde der betroffenen Menschen unvereinbaren personenbezogenen datenmäßigen Verknüpfung (von Tatverdächtigen bzw. Beschuldigten mit dem Begriff ,Täter ${ }^{6}$ ) ihrer Persönlichkeit.

Die bei der PKS-Erfassung entstehende dargestellte Datenverknüpfung hat Beunruhigung und Unbehagen auch in solchen Teilen der Bevölkerung hervorgerufen, die als loyale Staatsbürger und Beamte das Recht und die Pflicht des Staates respektieren, die für rationales und planmäßiges staatliches Handeln erforderlichen Informationen zu beschaffen. Diese PKS-Verfahrensweise kann bei Bürgern und Beamten Furcht vor einer unkontrollierbaren Persönlichkeitserfassung auslösen; denn der Einzelne wird durch die nicht sachgerechte Erhebung, Speicherung, Verwendung und Weitergabe seiner persönlichen Daten der freien Willensbestimmung beraubt und zum Gegenstand fremder Willensausübung. ${ }^{48}$

Beschränkungen dieses Rechts auf informationelle Selbstbestimmung sind nur im überwiegenden Allgemeininteresse zulässig; sie bedürfen einer (verfassungsgemäßen) gesetzlichen Grundlage, die dem rechtsstaatlichen Gebot der Normenklarheit entsprechen muss. ${ }^{49}$ Es liegt weder im überwiegenden Allgemeininteresse, verdächtige oder beschuldigte Personen rechtsstaatswidrig als ,Täter' zu bezeichnen bzw. zu kennzeichnen, noch dürfte eine entsprechende gesetzliche Grundlage die Unschuldsvermutung beschränken.

In den PKS-Richtlinien fehlen sachgerechte, klar definierte organisatorische und verfahrensrechtliche Vorkehrungen, um der Gefahr einer Persönlichkeitsrechts-Verletzung entgegen zu wirken. ${ }^{50}$ Somit konnte es zu den dargestellten persönlichkeitsfeindlichen Datenverknüpfung kommen, bei denen die Betroffenen zum bloßen (Informations-)Objekt staatlichen Handelns herabgewürdigt ${ }^{51}$ und als ,Täter' (vor-)verurteilt, stigmatisiert und sozial angeprangert werden.

Bei den verfahrensrechtlichen Anforderungen an derartige Einschränkungen wird zwar unterschieden zwischen personenbezogenen Daten, die in individualisierter, nicht anonymer Form erhoben und verarbeitet werden, und solchen, die für statistische Zwecke bestimmt sind. ${ }^{52}$ Diese Unterscheidung kann hier jedoch unberücksichtigt bleiben; denn vorliegend ist zumindest von einer personenbezogenen datenmäßigen Verknüpfung mit dem Begriff ,Täter' in den Erhebungs-, Speicherungs- und Verwendungs-Phasen auszugehen.

Im Übrigen ist auch die vom Grundgesetz selbst in Art. $73 \mathrm{Nr} .11$ vorgesehene und damit schutzwürdige amtliche Statistik gefährdet; denn für deren Funktionsfähigkeit ist ein möglichst hoher Grad an Genauigkeit und Wahrheitsgehalt der erhobenen Daten notwendig ${ }^{53}$, was hier nicht gegeben ist.

Eine Staatspraxis, die sich nicht um die Bildung des Vertrauens durch Offenlegung des Datenverarbeitungs-Prozesses bemühte und weiterhin derartige Datenverknüpfungen vornähme, würde bei Bürgern und Beamten Misstrauen gegenüber staatlichem Handeln ${ }^{54}$ provozieren.

\subsection{Verhältnismäßigkeit}

Die personenbezogene datenmäßige Verknüpfung von Tatverdächtigen bzw. Be- schuldigten mit dem Begriff ,Täter' missachtet den mit Verfassungsrang ausgestatteten Grundsatz der Verhältnismäßigkeit, der bereits aus dem Wesen der Grundrechte selbst folgt, die als Ausdruck des allgemeinen Freiheitsanspruchs des Staatsbürgers gegenüber dem Staat von der öffentlichen Gewalt jeweils nur insoweit beschränkt werden dürfen, als es zum Schutz öffentlicher Interessen unerlässlich ist ${ }^{55}$ : Die Maßnahme war zur Erreichung des angestrebten Zweckes ungeeignet, weil sie Unvereinbares miteinander verknüpfte (Tatverdächtige bzw. Beschuldigte mit dem Begriff ,Täter $\left.{ }^{\natural}\right)$, was in ihrer Tragweite für Bürger und Beamte unverständlich bleibt. Die Maßnahme war ferner nicht erforderlich; der mit ihr verbundene Eingriff stand auch außer Verhältnis zur Bedeutung der Sache und den vom Betroffenen hinzunehmenden Einbußen.

\subsection{Rechtsschutzgarantie}

Diese personenbezogene datenmäßige Verknüpfung von Tatverdächtigen bzw. Beschuldigten mit dem Begriff, Täter'verletzt ebenfalls die Rechtsschutzgarantie (Art. 19 Abs. 4 GG), die nicht nur das formelle Recht und die Möglichkeit, die Gerichte anzurufen, garantiert, sondern auch die Effektivität des Rechtsschutzes; der Bürger hat einen substanziellen Anspruch auf eine tatsächlich wirksame gerichtliche Kontrolle ${ }^{56}$ : Da durch die PKS-Verfahrensweise verhindert wird, dass der Betroffene (normalerweise) Kenntnis davon erlangt, wer wo über welche seiner personenbezogenen Daten in welcher Weise und zu welchen Zwecken verfügt, ist sein Rechtsschutz verfassungsrechtlich unzureichend. ${ }^{57}$ Einmal weitergegebene Daten sind im komplex verbundenen und vernetzten System der Polizei unterwegs, so dass sie nicht mehr zurückgehalten werden können, was die Rechtsschutzgarantie ebenfalls leerlaufen lässt. ${ }^{58}$

\section{Gebot einer konventions-, verfas- sungs- und grundrechtskonformen (Amts-)Sprache}

Seitdem der Bundesgesetzgeber in seiner Gesetzessprache in den $\$ \mathbb{S} 98$ a, $100 \mathrm{i}, 111$, 153 e, 163 d, 163 e, 163 f StPO, $\mathbb{4} 4$ BKAGesetz sowie $\mathbb{3} 31$ a BtM-Gesetz den hier verfassungsrechtlich anstößigen Begriff ,Täter' verwendet, ist es zu einem begriffsjuristischen Dammbruch gekommen. ${ }^{59}$

Nunmehr wurde diese den Rechtsstatus des Tatverdächtigen bzw. Beschuldigten missachtende Ausdrucksweise auch in Nord- 
rhein-Westfalen bei der Polizeilichen Kriminalstatistik etabliert:

In der Praxis geben Polizeibeamte personenbezogene Daten in die vom LZPD zur Verfügung gestellten Erfassungsmasken bzw. Datenfelder der IGVP- und PVP-Programme ein, die die Grundlage der PKS-Erfassung bilden. In diesen Programmen soll nunmehr - als Resultat der Eingabe - der Begriff ,Täter' (im weitesten Sinne) entfernt bzw. durch andere Begriffe ersetzt werden. Dies ist im Wesentlichen bis heute nicht geschehen, sollte aber in einem Rechtsstaat - der in ausgewogener Weise die Grundrechte der Bürger respektiert ${ }^{60}$ - eine Selbstverständlichkeit sein.

Jedoch soll der Begriff ,Täter‘ (i. w. S.) offensichtlich in den PKS-Richtlinien - für die das Landeskriminalamt federführend ist - weiterhin Bestand haben; ein (weiterer) Änderungsbedarf sei nicht erkennbar. ${ }^{61}$ Dies ist weder sachgerecht noch überzeugend. Gleichzeitig widersprechen diese LKA-Richtlinien den rechtsstaatlichen Grundsätzen. Da sie meines Erachtens die dogmatische Grundlage für die technischpraktische Umsetzung (Datenerfassung) der LZPD-Programme darstellen, müssten Richtlinien - und PKS-Vorstellung - erst recht durch eine auf äußerste Rechtsstaatlichkeit bedachte Sprache Orientierung und Sicherheit geben. Gerade bei der Polizei sollte jeder Anschein einer Vorverurteilung vermieden werden.

Im Interesse der europäischen Rechts- und Polizeikultur ${ }^{62}$ ist eine konventions-, verfassungs- und grundrechtskonforme, einheitliche Sprachregelung ${ }^{63}$ - auch in den Richtlinien, der Erfassung und der Vorstellung (in der Öffentlichkeit) der PKS - geradezu verpflichtend. Insbesondere Sicherheitsbehörden und Verfassungsorgane sollten einen Sensor für die unverfügbaren, abwägungsfesten Rechtspositionen - wie den Schutz der Menschenwürde und der Unschuldsvermutung ${ }^{64}$ - haben.

Deshalb möchte ich Sie bitten, Ihre Bewertung der Sach- und Rechtslage zu überdenken. (....).“

\section{Entscheidung des Landtages von Nordrhein-Westfalen}

Die Präsidentin des Landtages von Nordrhein-Westfalen ${ }^{65}$ teilte den vom Petitionsausschuss in seiner Sitzung vom 23.9.2008 gefassten Beschluss mit:
„Auch nach erneuter Prüfung der Sachund Rechtslage sieht der Petitionsausschuss keinen Anlass, seinen Beschluss vom 15.04.2008 zu ändern. Darüber hinaus hat der Petitionsausschuss die durchgeführten und geplanten Begriffsänderungen zur Kenntnis genommen. Der Petent erhält eine Kopie der ergänzenden Stellungnahme des Innenministeriums vom 29.08.2008.“

Die Stellungnahme des Innenministeriums des Landes Nordrhein-Westfalen ${ }^{66}$ hat folgenden Wortlaut:

„Den grundsätzlichen Ausführungen in meinem Schreiben vom 17.03.2008 - 42 - 13.05.03 - habe ich nichts hinzuzufügen. Darüber hinaus gehend nehme ich zu den neuen Vorbringen des Petenten folgt Stellung:

\section{Beamtenrechtliche Vorgaben}

Hierzu nimmt der Polizeipräsident Bonn wie folgt Stellung:

,Im Rahmen der erneuten Petition führt Herr Uwe Diercks auch an, dass seine ursprüngliche Petition vom 14.01.2008 von der Leiterin der Direktion Kriminalität nicht weitergeleitet worden sei. Herr Diercks hatte seine Petition vom 14.01.2008 parallel als Eingabe auf dem Dienstweg an das Innenministerium adressiert. Das als Petition zu wertende Schreiben mit gleichem Datum, welches Herr Diercks an die Vorsitzende des Petitionsausschusses gerichtet hatte, lag dem Petitionsausschuss bereits am 15.01.2008 vor. Das Innenministerium des Landes NRW hat diese Petition dem Landeskriminalamt als fachlich zuständige Behörde zur Prüfung und Stellungnahme zugeleitet. Eine Weiterleitung der inhaltlich identischen Eingabe war nicht erforderlich, da die von Herrn Diercks geschilderte Problematik ja bereits von der fachlich zuständigen Behörde (LKA) geprüft wurde und auch dem Innenministerium dieser Vorgang bereits vorlag.

Dieser Einschätzung des Polizeipräsidenten Bonn schließe ich mich an.

\section{Verwendung des Begriffs ,Täter}

Der Petent bemängelt, dass der Begriff ,Täter" noch nicht in allen Datenfeldbezeichnungen bzw. Katalogen der zur PKS-Erfassung eingesetzten DV-Anwendungen entfernt bzw. ersetzt wurde.

Dazu teilte das LZPD NRW mit, dass bisher Anpassungen der Eingabehinweise und der Maskenbeschriftungen erfolgten, soweit sie im Rahmen der Möglichkeiten des LZPD
NRW realisierbar waren. Dabei handele es sich im Einzelnen um folgende Änderungen:

Bereits mit der Einführung der DV-Anwendung PKS-online 2 zum 01.01.2008 wurde dort das Datenfeld ,Internationaler Straftäter' entsprechend der bundesweiten Regelung ersatzlos gestrichen. Im Datenfeld ,bereits polizeilich in Erscheinung getreten wurde der Katalogbegriff ,Wiederholungstäter' in der 30. KW gestrichen. Als Katalogbegriffe sind dort jetzt ,polizeilich in Erscheinung getreten' und ,nein/unbekannt erfassbar. Im Datenfeld ,Art der Tatbeteiligung' wurde in der 30. KW der Katalogbegriff ,Mittäter' durch den Katalogbegriff ,weiterer Tatverdächtiger` ersetzt.

Die Maskenbeschriftungen ,Täter ‘ und die Datenfeldbezeichnung ,Täter alleinhandelnd" in IGVP/PVP wurden - mit dem Update vom 04.08.2008 - in ,Tatverdächtiger' bzw. ,Tatverdächtiger alleinhandelnd' geändert. Das Datenfeld, Internationaler Straftäter' wurde ersatzlos entfernt. Die Katalogbegriffe ,Mittäter', ,Gehilfe‘ und ,Anstifter' werden in ,weiterer Tatverdächtiger ${ }^{`}$ umbenannt. Dazu sind Vereinbarungen auf KOOP-Ebene (DV-Kooperation mit den Ländern Bayern und Thüringen) herbeizuführen. Davon wird der Realisierungszeitpunkt im Wesentlichen bestimmt.

Die verbliebenen Beschriftungen in den PKS-relevanten Erfassungsmasken der Vorgangsverwaltung mit dem Begriff, Täter' gehören zum so genannten ,PVP-Kern', dessen Änderung nur mit Zustimmung der beiden Kooperationsländer Bayern und Thüringen möglich ist. Daher konnte das LZPD NRW eine kurzfristige Anpassung nicht vornehmen. Diese kann erst mit PVP-Release 6 Anfang Dezember 2008 durchgeführt werden. Damit sind die notwendigen Anpassungen mit Bezug zum PKS-Erfassungsvorgang initiiert.

Darüber hinaus habe ich festgestellt, dass auch andere Datenfelder, Kataloge und Formulare den Begriff ,Täter' enthalten. Ich werde prüfen, inwieweit auch in diesen Fällen der Begriff ersetzt werden kann.

Die Prüfung der über die dv-technischen Bezüge hinausgehenden Ausführungen des Petenten führte nicht $\mathrm{zu}$ anderen Ergebnissen als denen, die ich bereits mit meiner Stellungnahme vom 17.03.2008 dargelegt habe." 


\section{Schlussbetrachtung}

Wenn es stimmen sollte, was der angesehenste deutsche Justiz- und Kriminalreporter der Nachkriegszeit G. Mauz bereits im Jahre 1990 behauptete - ,wir stehen an einem Sarg, am Sarg der Unschuldsvermutung “67 -, dann wären die „Totengräber der Unschuldsvermutung " unter uns. Dann wäre unserem Rechtsstaat sowie der europäischen Rechts- und Polizeikultur ein unermesslicher Schaden zugefügt worden.

Trotz alledem hoffe ich sehr, dass sich G. Mauz in seiner Einschätzung geirrt haben möge, wenngleich die bekannten Tatsachen darauf hindeuten, dass er die Realität treffend beschrieben haben bzw. der Wahrheit sehr nahe gekommen sein dürfte.

Eine „Wiederbelebung“ der für verdächtige bzw. beschuldigte Personen einzufordernden Unschuldsvermutung erscheint meines Erachtens nur möglich, wenn sie von den Sicherheitsbehörden ${ }^{68}$, den Verfassungsorganen und der Presse nicht auf den Strafprozess beschränkt, sondern interdisziplinär ernst genommen und ihre Gültigkeit für das gesamte Rechtsleben anerkannt ${ }^{69}$ werden würde. Zudem müsste dies durch eine rechtsstaatlich faire ${ }^{70}$ und jeden Anschein einer unzulässigen Schuldzuweisung vermeidende $^{71}$ (Amts-) Sprache auch glaubhaft dokumentiert werden.

\section{Fußnoten:}

Der Beitrag gibt die persönliche Auffassung des Verfassers wieder und dokumentiert zugleich den Versuch, hinsichtlich der Verwendung des Begriffs „Täter“ in der Polizeilichen Kriminalstatistik (PKS) über den Petitionsausschuss des Landtages von Nordrhein-Westfalen und die Präsidentin des Landtages eine konventions-, verfassungs- und grundrechtskonforme sowie einheitliche Sprachregelung in den Richtlinien, der Erfassung und der Vorstellung (in der Öffentlichkeit) der PKS zu erreichen.

Mein herzlicher Dank gilt folgenden Rechtswissenschaftlern und Praktikern, die den in der Eingabe an den Petitionsausschuss des Landtages von Nordrhein-Westfalen bzw. in den Einwänden an die Präsidentin des Landtages geäußerten Bedenken zustimmten und / oder wertvolle Anregungen gaben: Professoren Dres. H. Achenbach, Osnabrück; W. Gropp, Gießen; W. Hassemer, Vizepräsident des Bundesverfassungsgerichts a. D., Karlsruhe / Frankfurt a. M.; K. Kühl, Tübingen; K. Marxen, Berlin; H.U. Paeffgen, Bonn; F.-C. Schroeder, Regensburg; R. Zaczyk, Bonn; Privatdozent Dr. K.-S. von Danwitz, Bonn; Wissenschaftliche Mitarbeiterinnen S. Oldenburg, für: Prof. Dr. B. Schlink, Berlin; Dr. E. Tophinke, Bern; Wissenschaftlicher Mitarbeiter Dr. J. Hohenhaus, Bonn; Rechtsanwälte Dr. K. Kokkinakis, Athen; Prof. Dr. Justizrat E. Müller, Saarbrücken; Prof. Dr. Justizrat F. Salditt, Neuwied; Polizeidirektor a. D. R. Wellenbeck, ehem. Leiter des Polizeiausbildungsinstituts Brühl / Aachen

1 Vgl.: Hassemer, F.-C.-Schroeder-FS (2006), S. 51, 61 („Absenkung der Eingriffsschwellen von Tatbestand oder Unschuldsvermutung “); SK-StPO/Paeffgen, 35. Lfg. (2004), EMRK, Art. 6 Rdnr. $190 \mathrm{ff}$. („Jedwede Schuldvermutung ist mit Art. 6 II unvereinbar.“); F.-C. Schroeder, NJW 2000, 2483 ff. (,weitere Einbruchstelle“); Diercks, AnwBl. 2002, 147 ff.

2 Eingabe vom 14.1.2008, I.3/14-P-2008-1093000

3 Siehe: Diercks, AnwBl. 2002, 147 ff.; ders., AnwBl. 1999, 311 ff., jeweils m.w.N.

4 BVerfGE 82, 106 (114)

5 Schreiben der Präsidentin des Landtages von Nordrhein-Westfalen vom 24.4.2008

6 Stellungnahme des Leitenden Ministerialrats Wesseler (Referatsleiter Organisation und Recht der Polizei) vom 17.3.2008, 42-13.05.03

7 Schreiben des Bonner Polizeipräsidenten W. Albers vom 11.4.2008, ZA 21.1-13.05.03

8 Einwände vom 23.6.2008

9 Vgl.: Hassemer, StV 2006, 321 (323 f.); ders., F.-C.-Schroeder-FS (2006), S. 51 (57 ff.); SKStPO/Paeffgen, 35. Lfg. (2004), EMRK, Art. 6 Rdnr. 192 ff.; ders., StV 1999, 625; ders., DRiZ 1998, 317 (320); Roxin, NStZ 1991, 153 ff.; F.-C. Schroeder, NJW 2000, 2483 ff.; Diercks, AnwBl. 2002, 147 ff.; ders., AnwBl. 1999, $311 \mathrm{ff}$.

10 Achenbach, Alternativkommentar zur StPO, Band 2, Teilband 1, 1992, \$ 111 Rdnr. 13, \$ 163 d Rdnr. 8; Artzt, Die verfahrensrechtliche Bedeutung polizeilicher Vorfeldermittlungen, Diss. Tübingen 1999, S. 138; Benfer, Rechtseingriffe von Polizei und Staatsanwaltschaft, 3. Aufl. (2005), S. 23 (zum „mutmaßlichen“ Täter); Bernsmann, NStZ 1989, 449 (459); Bernsmann / Jansen, StV 1998, 217; Binder, Rechtsprobleme des Einsatzes technischer Mittel gem. $\int \mathbb{S} 100$ c, d StPO und des Lauschangriffs, Diss. Bonn 1996, S. 22 f.; K. Braun, Handbuch der Gerichtsberichterstattung, 1994, S. 170; Dencker, KJ 1987, 36 (42); Eschke, Die Geltung der Unschuldsvermutung im Zivil- und Zivilverfahrensrecht, 2003, Diss. Potsdam 2002, S. 1, 10, 25, 36 ff.; Esser, Auf dem Weg zu einem europäischen Strafverfahrensrecht, Diss. Trier 2002, S. 100 f.; Fincke / Zoll, Strafrechtslehrertagung 2005, ZStW 117 (2005), S. 865 $(875,885)$; Gärditz, Strafprozess und Prävention, 2003, Diss. Bonn 2001, S. 58 („Täter“ als sprachliche Verfehlung); Grünwald, StV 1987, 453 (456); Hantschel, Jura 2001, 472 (474 Fn. 36); Hassemer, StV 2006, 321 (323 f.); ders., F.-C.-Schroeder-FS (2006), S. 51 (58); ders., in: Hassemer/Reemtsma, Verbrechensopfer. Gesetz und Gerechtigkeit, 2002, S. 52 ff.; ders., Strafen im Rechtsstaat, 2000, S. 266; ders., KJ 1992, 64 (69); ders., StV 1989, 72 (80); ders. StV 1986, 550 (552 f.); Hohenhaus, Die strafprozessuale Observation, Diss. Trier 2006, S. 221 ff., 241, 284; Hund, ZRP 1995, 334 (336); Jung, JZ 2003, 1096 (1097); Kargl, NStZ 2000, 8 (10); König, Kriminalistik 1998, 349 (351); Kokkinakis, Strafprozessuales operatives Vorgehen und strafrechtliches Rückwirkungsverbot, Diss. Frankfurt a. M. 2001, S. 71; Krauß, LüderssenFS (2002), S. 269 (271); Kühl, Müller-Dietz-FS (2001), S. 401 (406 f.); ders., Unschuldsvermutung, Freispruch und Einstellung, 1983, Habil. Schrift Bielefeld 1981, S. 109; ders., in: Lackner / Kühl, Strafgesetzbuch, 26. Aufl. (2007), \$193 Rdnr. 11; Kühne, Strafprozessrecht, 7. Aufl. (2006), S. 185; Lagodny, 20. Strafverteidigertag, 1996, S. 117 (127); Lamprecht, DRiZ 1989, 32; Lisken, in: Lisken / Denninger, Handbuch des Polizeirechts, 3. Aufl. (2001), S. 925 Fn. 37; Maiwald, GA 2005, 339, 342 f. (zum „vermutlichen“ Täter); Marxen, Straftatsystem und Strafprozess, 1984, Habil.-Schrift Frankfurt a. M. 1982, S. 345; ders., GA 1980, 365 (379); Meyer-Ladewig, Nomos-Kommentar, 2. Aufl. (2006), EMRK, Art. 6 Rdnr. 86; E. Müller, Beiträge zum Strafprozessrecht (1969 - 2001), 2003, S. 79; ders., Müller-Dietz-FS
(2001), S. 567 (569 f.); Neuling, Inquisition durch Information, 2005, Diss. Berlin 2004, S. 164, 247; Nimtz, Die strafprozessuale Observation nach dem Strafverfahrensänderungsgesetz 1999, 2003, Diss. Köln 2002, S. 27, 116 ; Nitz, Einsatzbedingte Straftaten Verdeckter Ermittler, Diss. Hannover 1997, S. 121 f.; Ott, Verdeckte Ermittlungen im Strafprozess, Diss. Berlin, 2008, S. 194 Fn. 892; SK-StPO/Paeffgen, 35. Lfg. (2004), EMRK, Art. 6 Rdnr. 193 , 202; ders., StV 1999, 625; ders., DRiZ 1998 , 317 (320); Prinz / Peters, Medienrecht, 1999 S. 239 f.; Püschel, StraFo 2006, 261; Raum / Palm, JZ 1994, 447 (452 Fn. 45); Riepl, Informationelle Selbstbestimmung im Strafverfahren, 1998, Diss. Tübingen 1994, S. 55; Rieß, StraFo 1999, 1 (9); KK-Schädler, 6. Aufl. (2008), MRK, Art. 6 Rdnr. 44; F.-C. Schroeder, Strafprozessrecht, 4. Aufl. (2007), Rdnr. 368; ders., GA 2005, 73; ders., NJW 2000, 2483 ff. (hinzufügend: „Zielbeschreibungen und Anknüpfungen an den mutmaßlichen Täter sind auch in den Vorschriften über die Regelung des Ermittlungsverfahrens zulässig. ... Eine Formulierung wie ,Das Strafverfahren dient dem Zweck, den Täter der Bestrafung zuzuführen wäre durchaus möglich. “); U. Schulz, Die rechtlichen Auswirkungen von Medienberichterstattung auf Strafverfahren, 2002, Diss. Frankfurt a. M. 2001, S. 30, 140; Tophinke, Das Grundrecht der Unschuldsvermutung, 2000, Diss. Bern 1999, S. 139, 356, 363 ff., 395 ff., 468 f.; Wohlers, GA 2005, 11 (12); Zaczyk, StV 1993 490 (491); Zeller, Zwischen Vorverurteilung und Justizkritik, Diss. Bern 1998, S. 60, 94, 98 f., 265. Ebenso: Diercks, AnwBl. 2002, 147 ff. (149); ders., AnwBl. 1999, 311 ff. (insbes. 316 Fn. 78 )

11 Landeskriminalamt (LKA) Nordrhein-Westfalen, SG 32.2, Richtlinien für die Führung der Polizeilichen Kriminalstatistik i. d. F. vom 1.1.2008

12 Innenministerium Nordrhein-Westfalen, Runderlass vom 1.1.2003

13 Landesamt für Zentrale Polizeiliche Dienste (LZPD) Nordrhein-Westfalen, InteGrationsVerfahren-Polizei, Eingabehinweise, PKS-Meldung aus PVP, Stand: 11/2007

14 LKA NRW (s. Fn. 11), S. 6 ff.

15 LKA NRW (s. Fn. 11), S. 8

16 Hassemer, AnwBl. 2008, 413, 420 (spricht hinzufügend von „möglichen Tätern“). Vgl.: Tophinke, Das Grundrecht der Unschuldsvermutung (Fn. 10), S. 425 („Angesichts der Fehleranfälligkeit menschlicher und namentlich auch richterlicher Erkenntnis muss daran festgehalten werden, dass die Unschuldsvermutung erst durch eine rechtskräftige Verurteilung umgestoßen werden kann.“)

17 Zum Begriff des „Mitbeschuldigten“ bereits: BGHSt 10, 8 (10 f.)

18 LZPD NRW (s. Fn. 13), S. 3

19 LZPD NRW (s. Fn. 13), S. 5

20 LZPD NRW (s. Fn. 13), S. 5

21 LZPD NRW (s. Fn. 13), S. 6

22 LZPD NRW (s. Fn. 13), S. 8

23 LKA NRW (s. Fn. 11), S. 24

24 LKA NRW (s. Fn. 11), S. 30

25 LKA NRW (s. Fn. 11), S. 31

26 LKA NRW (s. Fn. 11), S. 30, 32 f. Vgl. zur Amtshilfe im Bereich der Inneren Sicherheit: Schlink, Die Amtshilfe. Ein Beitrag zu einer Lehre von der Gewaltenteilung in der Verwaltung, 1982, Habil.-Schrift Freiburg 1981, S. 261 ff.; ders., Jura 1999, 169 (171, zu den Aufgaben der Exekutive)

27 Innenminister I. Wolf, (Bonner) General-Anzeiger vom 4.3.2008, S. 1, sowie Presse-Information des Innenministeriums des Landes Nordrhein-Westfalen vom 3.3.2008

28 Polizeipräsident W. Albers und Leitende Kriminaldirektorin G. Hewer-Brösch, (Bonner) General-Anzeiger vom 4.3.2008, S. 6 
29 Innenminister I. Wolf (s. Fn. 27). Der (Bonner) General-Anzeiger berichtete am 24./25.5.2008 (S. 1) auch über die Vorstellung der Polizeilichen Kriminalstatistik 2007 durch Bundesinnenminister W. Schäuble, der (verfassungskonform) von „tatverdächtigen Jugendlichen “ und „heranwachsenden Verdächtigen“ sprach (s. BMI, PKS 2007, S. 9 f.; NJ 2008, 300 ff.). Bei den Pressemitteilungen zur Veröffentlichung der PKS 2007 wird für Sachsen-Anhalt ebenfalls (verfassungskonform) von „Jungtatverdächtigen“ gesprochen, während für Mecklenburg-Vorpommern über „Jungtäter“, "Intensivtäter“ und „Straftäter“ berichtet wird (NJ 2008, 253 ff.)

30 Leitende Kriminaldirektorin G. Hewer-Brösch (s. Fn. 28), ebenso im (Bonner) General-Anzeiger vom 2.10.2007, S. 6

31 Vgl.: Benda, ZRP 2008, 63 (zum „Richteramtsbonus“); ders., Zeidler-FS (1987), S. 43 ff.

32 So auch das LKA NRW (s. Fn. 11), S. 6. Allgemein zum strafrechtlichen „Täter“-Begriff: F.-C. Schroeder, Der Täter hinter dem Täter, Diss. München 1962, S. 13, 58 ff., 221 f.; Welzel, Das Deutsche Strafrecht, 11. Aufl. (1969), $\mathbb{S} 7 \mathrm{I}, \mathbb{S} 8 \mathrm{I}, \mathbb{} 10$

33 Justizministerin R. Müller-Piepenkötter, (Bonner) General-Anzeiger vom 27.3.2008, S. 5 (zu verurteilten Tätern)

34 Vgl.: Frowein, FS 50 Jahre BVerfG (2001), S. 209 (218 ff.); Limbach, AnwBl. 2002, 454 (457); dies., NJW 2001, 2913; Mahrenholz, in: Badura / Scholz, Verfassungsgerichtsbarkeit und Gesetzgebung, 1998, S. 23 (29 ff.); Paeffgen, Küper-FS (2007), S. 389 ff.; ders., ZStW 118 (2006), S. 275 (354); ders., DahsFS (2005), S. 143 ff.; ders., StV 2002, 336 ff.; Schlink, JZ 2007, 157 ff. (zum Verhältnis des EGMR zum BVerfG); von Weber, ZStW 65 (1953), S. 334 ff.; Wildhaber, EuGRZ 2002, 569 ff.; Diercks, (Bonner) General-Anzeiger vom 8.10.1991, S. 14

35 Vgl.: Hirsch, Nehm-FS (2006), S. 61 (64 ff.); Papier, EuGRZ 2006, 1; Stuckenberg, StV 2007, 655, 663 („Denn die Beachtung der EMRK in der Auslegung des EGMR durch deutsche Gerichte, die das BVerfG im Görgülü-Beschluss als Ausfluss der Bindung an Recht und Gesetz (Art. 20 Abs. 3 GG) nachdrücklich angemahnt hat, lässt im Gebiet des Strafverfahrens immer noch zu wünschen übrig.")

36 BVerfGE 65, 1 (60) „Volkszählungsgesetz“

37 LKA NRW (s. Fn. 11), S. 6, 30; LZPD NRW (s. Fn. 13), S. 3

38 Vgl.: A. Arndt, NJW 1960, 1191 (1193); Diercks, AnwBl. 2002, 147 (151 ff.); ders., AnwBl. 1987, 154 (168 ff.); Geppert, F.-C.Schroeder-FS (2006), S. 675 (679 ff.); Groß, Dahs-FS (2005), S. 249 ff.; Hassemer, in: Pieth / Seelmann, Prozessuales Denken als Innovationsanreiz für das materielle Strafrecht, 2006, S. 9 (20 ff.); ders., JuS 1991, 256 f.; E. Müller, Hamm-FS (2008), S. 489 ff.; Paeffgen, SeebodeFS (2008), S. 245 ff.; ders., StV 2007, 648 ff.; ders., NK-Paeffgen, 2. Aufl. (2005), Vor $\$ 32$ Rdnr. 75; ders., GA 2003, 647 ff.; ders., in: Wolter / Schenke, Zeugnisverweigerungsrechte bei (verdeckten) Ermittlungsmaßnahmen, 2002, S. 215 (218 ff.); ders., Vorüberlegungen zu einer Dogmatik des UntersuchungshaftRechts, 1986, Habil.-Schrift Mainz 1982, S. 8 (Prozessrecht als „angewandtes Verfassungsrecht“); Rieß, JR 2006, 269 ff.; ders., StraFo 1999, 1 (9); ders., NJW 1998, 3240 (3243); Roxin, Strafverfahrensrecht, 25. Aufl. (1998), \ 2 Rdnr. 1 (Strafverfahrensrechts als "Seismograph der Staatsverfassung "); Salditt, in: Widmaier, Münchener Anwalts-Handbuch, Strafverteidigung, 2006, $\mathbb{1} 1 \mathrm{Rdnr}$. 89 ff.; F.-C. Schroeder, Roxin-FS (2001), S. 33 (41); Tolksdorf, Grünwald-FS (1999), S. 731 ff.; Wohlers, Rudolphi-FS (2004), S. 713 (715 ff.); Zaczyk,
Otto-FS (2007), S. 191 ff.; ders., StV 1993, 490 (492)

39 Roxin, JR 2008, 16 ff.; Eisele, JR 2004, 12 ff.; Krack, Rehabilitierung des Beschuldigten im Strafverfahren, 2002, Habil.-Schrift Göttingen 2001, S. 102 f.; Lindner, StV 2008, 210 ff.; BGHSt 37, 48 ff.; BGH, JA 2008, 151 f., m. Anm. von Heintschel-Heinegg

40 Zur Unschuldsvermutung, die nur durch eine rechtskräftige strafgerichtliche Verurteilung widerlegt werden kann: BVerfGE 35, 202 (232); 74, 358 (371); Blumenstein, Der Widerruf der Strafaussetzung zur Bewährung wegen der Begehung einer neuen Straftat nach $\$ 56 \mathrm{f}$ Abs. 1 Satz 1 Nr. 1 StGB, Diss. Gießen 1995, S. 137 f.; Dahs, Handbuch des Strafverteidigers, 7. Aufl. (2005), Rdnr. 1; Gerhardt / Steffen, Kleiner Knigge des Presserechts, 2. Aufl. (1997), S. 48; Hassemer, in: Hassemer / Reemtsma (Fn. 10), S. 53; Krauß, Lüderssen-FS (2002), S. 269 (271); Kühl, Meyer-Gossner-FS (2001), S. 715 (730 f.); ders., Hubmann-FS (1985), S. 241 (251); ders., NJW 1980, 806 (809); ders., JR 1978, 94 (96 ff.); Mahrenholz, in: Mahrenholz / Hilf / Klein, Entwicklung der Menschenrechte innerhalb der Staaten des Europarates, 1987, S. 73 (76); Nierwetberg, NJW 1989, 1978 f.; Paeffgen, Vorüberlegungen (Fn. 38), S. 51; ders., Haftgründe, Haftdauer und Haftprüfung, in: Viertes deutsch-polnisches Kolloquium über Strafrecht und Kriminologie, 1991, S. 113 Fn. 85; ders., DRiZ 1998, 317 (318); Peukert, EuGRZ 1980, 247 (259); Pfeiffer, Geiß-FS (2000), S. 147 (152); Stuckenberg, ZStW 111 (1999), S. 422 (445); ders., Untersuchungen zur Unschuldsvermutung, 1998, Diss. Bonn 1997, S. 85; Tophinke, Das Grundrecht der Unschuldsvermutung (Fn. 10), S. 363 ff., $425,468 \mathrm{f}$.

41 Zum „verzerrten Bild vom Straftäter, der Kriminalität und der Struktur der Delinquenz": Jung, in: Kleines Kriminologisches Wörterbuch, 3. Aufl. (1993), S. 345 (346); zustimmend: Hassemer, Burgstaller-FS (2004), S. 325 (327, 329). Vgl.: OLG Celle, NJW 2004, 1461 f. (zur schuldhaften Amtspflichtverletzung nach $\mathbb{8} 839$ Abs. 1 BGB i.V.m. Art. 34 GG, wenn Polizeibeamte bei Pressekonferenzen statt von einem begründeten Tatverdacht von einem Schuldnachweis - als „Täter“ überführt - sprechen)

42 BVerfGE 82, 106 (117). Vgl.: Diercks, AnwBl. 2002, 147 (152 m. w. N.)

43 Allgemein zur Unschuldsvermutung: EGMR, NJW 2006, 1645; NJW 2006, 1113 f.; StV 2003, 82; EuGRZ 1987, 399 (404), Abw. M. Cremona; EuGRZ 1983, 475 (479); BVerfGE 19, 342 (347 f.); 20, 144 (147); 22, 254 (265); 25, 327 (331); 35, 202 (232) „Lebach“; 35, 311 (319 f.); 71, 206 (216 f.); 74, 358 (370); 82, 106, 122 (124 f.), Abw. M. Mahrenholz; 110, 1 (22 f.); BVerfG, NJW 2002, 3231 f.; NJW 1994, 377; NJW 1992, 2011; NJW 1992, 1612; NJW 1991, 1530 ff.; NJW 1988, 1715 f.; BGHSt 14, 358 (364); 24, 125 (131); Achenbach, in: Historisches Wörterbuch der Philosophie, Band 11, 2001, Sp. 266 f.; A. Arndt, NJW 1966, 869 ff.; ders., NJW 1960, 1191 f.; Benda, AnwBl. 2005, 602 ff.; Dahs, NStZ 1986, 563; ders., NJW 1976, 2145 f.; K. S. von Danwitz, Staatliche Straftatbeteiligung, 2005, Habil.-Schrift Bonn 2001, S. 85 ff.; Demko, HRRS 2007, 286 ff.; Dencker, JZ 1973, 144 (150); Gaede, Fairness als Teilhabe - Das Recht auf konkrete und wirksame Teilhabe durch Verteidigung gemäß Art. 6 EMRK, 2007, Diss. Zürich 2005, S. 228 ff.; Gropp, JZ 1991, 804 ff.; Hoffmann, Die Polizei 2006, 170 (171 f.); Kieschke, Die Praxis des Europäischen Gerichtshofs für Menschenrechte und ihre Auswirkungen auf das deutsche Strafverfahrensrecht, 2003, Diss. Halle-Wittenberg, 2002, S. 229 ff.; Kühl, Jung-FS (2007), S. 433 (441 f.); ders., NJW 1988, 3233 ff.; ders.,
Hubmann-FS (1985), S. 241 (251); ders., NJW 1984, 1264 (1267); ders., JR 1978, 94; Lilie, F.-C.-Schroeder-FS (2006), S. 829 (835, 839 f.); Limbach, EuGRZ 2000, 417 f.; Löwe-Rosenberg/Gollwitzer, 25. Aufl. (2005), MRK, Art. 6 Rdnr. 106 ff.; Mauz, in: Unschuldsvermutung in der Mediengesellschaft, 1990, S. 40, 44 (behauptet: „wir stehen ... am Sarg der Unschuldsvermutung “); E. Müller, Koch-Festgabe (1989), S. 191 (193); Neubacher, GA 2004, 402 ff. Paeffgen, Fezer-FS (2008), S. 35 ff.; ders., StV 2007, 487 (492 f.); ders., NStZ 2007, 142 (147 f.); SK-StPO/Paeffgen, 54. Lfg. (2007) Vor $\mathbb{1 1 2}$ Rdnr. 21 ff.; ders., StV 2004, 160 (162 ff.); ders., Vorüberlegungen (Fn. 38), S. 42 ff., 62 ff., 77; Paeffgen / Seebode, ZRP 1999, 524 (525 f.); Roxin, StPO (Beck-Texte), 43. Aufl. (2008), Einführung, S. IX; ders. NStZ 1991, 153 (156); Salditt, 4. Petersberger Tage 2007; F.-C. Schroeder, GA 2003, 293; ders., JZ 2000, 409 f.; ders., in: Wolter (Hrsg.), 140 Jahre Goltdammer's Archiv für Strafrecht, 1993, 205 (209); L. Schulz, Normiertes Misstrauen. Der Verdacht im Strafverfahren, 2001, Habil.-Schrift Frankfurt a. M. 1997, S. 483, 486; ders., GA 2001, 226 f.; Soehring, Vorverurteilung durch die Presse. Der publizistische Verstoß gegen die Unschuldsvermutung, Diss. Hamburg 1999, S. 27, 45, 58 ff., 238; Stuckenberg, Hilger-Festgabe (2003), S. 25 (40 ff.); Szczekalla, in: Heselhaus / Nowak, Handbuch der Europäischen Grundrechte, 2006, \$5 52 Rdnr. 3

44 Britz, Fernsehaufnahmen im Gerichtssaal, Diss. Saarbrücken 1999, S. 255; Diercks, AnwBl. 2002, 147 (150); ders., AnwBl. 1999, 311 (314 f.); Höpfel, Staatsanwalt und Unschuldsvermutung, 1988, Habil.-Schrift Innsbruck 1986, S. 21

45 Vgl.: BVerfGE 65, 1 (43, 48) „Volkszählungsgesetz"

46 BVerfGE 65, 1 (41) „Volkszählungsgesetz“

47 BVerfGE 65, 1 (43) „Volkszählungsgesetz“. Vgl.: Paeffgen, Hilger-Festgabe (2003), S. 153 ff. (zu den Kompetenzen zur (präventiven und repressiven) Datenübermittlung); ders., Rieß-FS (2002), S. 413 ff. (zur heimlichen Informationserhebung)

48 Vgl.: BVerfGE 65, 1 (4, 17) „Volkszählungsgesetz"

49 BVerfGE 65, 1 (44) „Volkszählungsgesetz“

50 Vgl.: BVerfGE 65, 1 „Volkszählungsgesetz“

51 Vgl.: BVerfGE 57, 250 (275); Eschke, Die Geltung der Unschuldsvermutung (Fn. 10), S. 31; Radbruch, Sauer-FS (1949), S. 121 (126); Schilling, Illegale Beweise, 2004, Diss. Frankfurt a. M. 2003, S. 234; Wulf, Strafprozessuale und kriminalpraktische Fragen der polizeilichen Beschuldigtenvernehmung auf der Grundlage empirischer Untersuchungen, Diss. Hamburg 1984, S. 7 ff., 129

52 BVerfGE 65, 1 „Volkszählungsgesetz “

53 BVerfGE 65, 1 (50) „Volkszählungsgesetz“

54 Vgl.: BVerfGE 65, 1 (50) „Volkszählungsgesetz"

55 Vgl.: BVerfGE 19, 342 (348); 65, 1 (44) „Volkszählungsgesetz"

56 Vgl.: BVerfGE 53, 115 (127 f.); 65, 1 (70) „Volkszählungsgesetz“

57 Vgl.: BVerfGE 65, 1 (70) „Volkszählungsgesetz"

58 Vgl.: BVerfGE 65, 1 (20 f.) „Volkszählungsgesetz"

59 Vgl.: Hassemer, F-C.-Schroeder-FS (2006), S. 51, 61 („Absenkung der Eingriffsschwellen von Tatbestand oder Unschuldsvermutung“); F.-C. Schroeder, NJW 2000, 2483 ff. („weitere Einbruchstelle“); Marxen / Tiemann, ZIS 2008, 188 ff. (äußern verfassungsrechtliche Bedenken zum geplanten Gesetzesvorhaben - \$ 362 Nr. 5 StPO; BR-Drs. 655/07 - der Wiederaufnahme zu Ungunsten des Angeklagten und dokumentieren, wie notwendig das Beharren auf einer 
umfassenden Respektierung der Unschuldsvermutung ist; denn das Gesetzesvorhaben zielt auf eine Erweiterung der Möglichkeit, die Wiederaufnahme eines rechtskräftig abgeschlossenen Verfahrens zu Ungunsten des Freigesprochenen zu betreiben. Im Zusammenhang mit einer rechtskräftig freigesprochenen Person wird hier von „Täter" und „Täterschaft" gesprochen.); Diercks, AnwBl. 2002, $147 \mathrm{ff}$.

60 Vgl.: Schünemann, ZIS 2007, 528

61 Der nachfolgenden Argumentation im Beschluss des Petitionsausschusses kann aus den zuvor genannten Gründen nicht gefolgt werden: „In der polizeilichen Kriminalstatistik werden die Begriffe ,Täter', ,Mittäter' bzw. ,Einzeltäter zur Erläuterung kriminologischer oder strafrechtlicher Definitionen, zur Erklärung von Grenzfällen und zur Verdeutlichung der kriminalstatistischen Zuordnung von Sachverhalten genutzt. Sie dienen, ohne jeden Bezug zu konkreten Personen, lediglich zur Klarstellung von Tatbeständen."

62 Zur (europäischen) Rechtskultur: Dilcher, NJW 1998, 3690 (3692); Gläser, Die Sprache in der Rechtsprechung des Reichsgerichts in Strafsachen, Diss. Bonn 1955, S. 3, 6; Hamacher, AnwBl. 6/2008, S. I; Hassemer, AnwBl. 2008, $413 \mathrm{ff}$. (zur "Sicherheitsorientierung unserer derzeitigen Rechtskultur“); ders., Der Spiegel, Nr. 12/2008, S. 38 ff.; ders., in: Höffe, Vernunft oder Macht, 2006, S. 171 ff., 181 (Der Schutz der Menschenwürde sei ein Pfeiler der Leitkultur des „Alten Europa“.); ders., StraFo 2005, 312 (317 f.); ders., Strafen im Rechtsstaat, 2000, S. 265 f.; ders., KJ 1992, 64 (67); Höland, in: Renzikowski, Die EMRK im Privat-, Strafund Öffentlichen Recht, 2004, S. 11 ff.; Hoffmann-Riem, EuGRZ 2002, 473 (479); Jung, JZ 2003, 1096, 1099 („Prozesskultur“); Kerscher, DRiZ 1983, 439 (442); Kirchhof, NJW 2002, 2760 f.; Köhler, Der Spiegel, Nr. 1/2007, S. $22 \mathrm{f}$. (regt an, dass wir ,einen Blick auf die Gesetzgebungskultur werfen sollten, wenn wir uns auf die Suche nach den Ursachen für die Distanz zwischen Bürgern und Politik machen. Gründlichkeit und Transparenz sollten jedenfalls selbstverständliche Standards in der Gesetzgebung sein.“); Kokkinakis, Strafprozessuales operatives Vorgehen (Fn. 10), S. 266 (Unschuldsvermutung als „Rechtsinstitut mit kulturkreisübergreifender und intertemporaler Geltung"); Lamprecht, NJW 2007, 2744 (2746); Paeffgen, ZStW 118 (2006), S. 275 (357); Roxin, Strafverfahrensrecht, 25. Aufl. (1998), \ 2 Rdnr. 1; Seher, ZStW 118 (2006), S. 101, 122 (zur „judikativen Sprachkultur“ im Zusammenhang mit der Unschuldsvermutung); Sommer, StraFo 2002, 309, 311, 315 (zur transnationalen und europäischen Rechtskultur); Uerpmann, Die Europäische Menschenrechtskonvention und die deutsche Rechtsprechung, 1993, Diss. Berlin 1991, S. 132, 245 f.; Wohlers, GA 2005, 11 (26, 35). Siehe auch: A. Arndt, Gesammelte juristische Schriften, 1976; G. Mauz, Die großen Prozesse der Bundesrepublik Deutschland, 2005; R. Schmid, Letzter Unwille, 1984; ders., Das Unbehagen an der Justiz, 1975; ders., Einwände, 1965

63 Vgl.: BVerfGE 82, 106, 122 (124 f.), Abw. M. Mahrenholz; A. Arndt, NJW 1960, 1191 (1192 f.); Beradt, Der Deutsche Richter, 1979, S. 219 ff.; Y. Braun, Medienberichterstattung über Strafverfahren im deutschen und englischen Recht, Diss. Gießen 1997, S. 108; Bornkamm, Pressefreiheit und Fairness des Strafverfahrens, Diss. Freiburg 1980, S. 223; Diercks, AnwBl. 1987, 154 (168 ff.); ders., Die Polizei 1985, 161 (168); ders., Die Polizei 1985, 256; Ennuschat, DVBl. 2004, 986 ff.; Gläser, Die Sprache in der Rechtsprechung (Fn. 62), S. 1 ff., 37 f., 40 ff., 90 ff., 101 ff.; Grabenwarter, Europäische Menschenrechtskonvention, 2003, $\mathbb{2} 24$ Rdnr. 78 (82); Gossfeld,
Unsere Sprache: Die Sicht der Juristen, 1990, S. 7, 31 ff., 40 ff.; Hassemer, F.-C.-SchroederFS (2006), S. 51, 57 f. („Der Strafgesetzgeber läßt sich vom aggressiven und punitiven Stil des kriminalpolitischen Diskurses beeindrucken, gibt die ihm angemessene Zurückhaltung auf und bedient sich zunehmend einer Rhetorik der ,Bekämpfung ‘ von Kriminalität, flankiert von einer schlampigen Redeweise, die schon in Regelungen des Ermittlungsverfahrens schlicht von ,Tätern' spricht, als hätte die Unschuldsvermutung nicht auch Konsequenzen für die Art und Weise, wie sich ein Verfassungsorgan in den Strafgesetzen ausdrückt.“); ders., StV 2006, 321, 323 („Das Strafrecht ... wird ausgerüstet als Kampfinstrument. Die derzeitige gängige Rhetorik des Strafgesetzgebers, die auch bei scheinbar randständigen Gesetzen nicht auf die militante Überschrift ,Bekämpfung' verzichten mag und die auch im Strafprozessrecht trotz der Unschuldsvermutung ungeniert von Tätern spricht, sind dafür nur eine Grundierung“); ders., in: Sieber, Internationale Organisierte Kriminalität, 1997, S. 213 (218); Hohenhaus, Die strafprozessuale Observation (Fn. 10), S. 221 ff., 241; Hirschberg, Das Fehlurteil im Strafprozess, 1960, S. 96 ff.; Isensee, in: Ipsen / Rengeling, Verfassungsrecht im Wandel, 1995, S. $571 \mathrm{ff}$.; Keller, ZRP 2002, $524 \mathrm{ff}$.; Kirchhof, NJW 2002, 2760; Kühl, ZStW 100 (1988), S. 406 (414 f.); ders., Unschuldsvermutung (Fn. 10), S. 124, 133; ders., NStZ 1981, 114 f.; ders., NJW 1980, 806 (810); Kühne, Schneider-FS (1998), S. 3; Ludwig, Persönlichkeitsrechtsverletzungen durch identifizierende Presseverlautbarungen der Staatsanwaltschaft, Diss. Bonn 1998, S. 144; Lüke, NJW 1995, 1067 f.; Marxen, Schneider-FS (1998), S. 297 (302); ders., Straftatsystem (Fn. 10), S. 345; ders., GA 1980, 365 (373); Mertin, ZRP 2004, 266 ff.; E. Müller, Müller-Dietz-FS (2001), S. 567 ff.; Paeffgen, JR 1999, 89 (96); ders., NJ 1996, 455; ders., Vorüberlegungen (Fn. 38), S. 54, 77; Redeker, ZRP 2004, 160 ff.; ders., NJW 2002, $2756 \mathrm{ff}$; R. Schmid, Einwände (Fn. 62), S. 132 ff.; Schmidhäuser, Dünnebier-FS (1982), S. 407 ff.; Schnapp, JZ 2004, 473 ff.; F.-C. Schroeder, NJW 2000, 2483 f.; ders., Zipf-Gedächtnisschrift (1999), S. 153; ders., Peters-FS (1974), S. 411 (418, 421); Simon, Die Beschuldigtenrechte nach Art. 6 Abs. 3 EMRK, Diss. Tübingen 1998, S. 227; Tophinke, Das Grundrecht der Unschuldsvermutung (Fn. 10), S. 139 ff., 365, 395 ff., 468; Wagner, Strafprozessführung über Medien, 1987, S. 48 f.; Walter, JR 2007, 61 ff.; Westerdiek, EuGRZ 1987, 393 f. (397); Wildhaber, Der Spiegel, Nr. 47/2004, S. 50, 54 (zur „,angelsächsischen Präzision und Klarheit in Gerichtsurteilen “)

64 Vgl.: Hassemer, F.-C.-Schroeder-FS (2006), S. 51 (58, 60 ff.); SK-StPO/Paeffgen, 54. Lfg. (2007), Vor $\mathbb{} 112$ Rdnr. 26

65 Schreiben der Präsidentin des Landtages von Nordrhein-Westfalen vom 25.9.2008, I.3/14-P2008-10930-01

66 Stellungnahme des Leitenden Ministerialrats Wesseler (Referatsleiter Organisation und Recht der Polizei) vom 29.8.2008, 42-13.05.03

67 G. Mauz, in: Unschuldsvermutung in der Mediengesellschaft, 1990, S. 40 (44)

68 Bereits 1971 (!) äußerte schon einmal ein Bonner Polizeibeamter erhebliche Bedenken an der PKS: Ritgen, Die Polizeiliche Kriminalstatistik - unter besonderer Berücksichtigung ihrer Fehlerquellen, Diss. Bonn 1971, S. 29, 31, 34 (kritisiert, dass im Stadium des (kriminal-)polizeilichen Ermittlungsverfahrens von einer „Überführung des Täters“ jedenfalls nicht die Rede sein dürfte und hier der Umgang mit dem Begriff „Täter“ erheblichen Bedenken begegne. Aus dem „Erfordernis unzweideutiger Begriffsinhalte im statistischen Bereich" resultiert sein Begriffsvorschlag „Tatperson“ für eine neue
PKS-Regelung.). Vgl.: Doka, Die Kontrolle von Vertrauenspersonen im Strafprozess, 2008, Diss. Bielefeld 2007, S. 374, 381 (zur eingeschränkten Unschuldsvermutung, die sich nicht mehr an rechtsstaatlichen Grundsätzen orientiere: "Zugespitzt hat ein Polizeibeamter die in der Praxis vorherrschende Auffassung formuliert, er habe noch keine Telefonüberwachung erlebt, in der sich nachher die Unschuld des Tatverdächtigen herausgestellt habe. Diese Ansicht hat jedoch zur Folge, dass in bestimmten Milieus eine Art Umkehr der Beweislast stattfindet und der Tatverdächtige als schuldig gilt bis zum Beweis des Gegenteils. “); Artkämper, Polizeibeamte als Zeugen vor Gericht, 2007, S. 3 (Gemeinsames Verfahrensziel von Polizei, Staatsanwaltschaft und Gericht sei - bis zur Rechtskraft des Urteils - „die Verurteilung des Täters, ggf. die Freisprechung eines zu Unrecht in Verdacht geratenen Beschuldigten."); Förster, Der Täterschwund zwischen der Polizeilichen Kriminalstatistik und der Strafverfolgungsstatistik, Diss. Kiel 1986, S. 90 f. („Während die Polizei gegen den Verdächtigen unter der Annahme ermittelt, er sei der Täter, ist die Justiz eher von der Unschuldsvermutung geprägt."); Heger, GA 2009 , 45 (47 f.); Lindner, AöR 133 (2008), S. 235 (240 f.). Die Presse berichtete (unwidersprochen) über das öffentlich geäußerte Vorhaben des Innenministers I. Wolf, wonach die Polize in NRW gezielt gegen ,jugendliche Gewalttäter" vorgehen wolle. Knapp 1.500 Kinder und junge Heranwachsende würden derzeit von der Polizei als „Intensivtäter“ eingestuft (Bonner General-Anzeiger, 30.7.2008, S. 5; s. PresseInformation des IM NRW vom 3.3.2008 zur PKS-Vorstellung, S. 4 ff.). Die Erfassung von schuldunfähigen Kindern als Tatverdächtige in der PKS sollte unterbleiben. Entsprechend verfährt etwa die Schweiz, um junge Menschen - die sich noch entwickeln und noch erzogen werden - vor einer Stigmatisierung als „Kriminelle“ zu schützen (Kunz, Kriminologie, 4. Aufl. (2004), \$ 27 Rdnr. 47 m.w.N.).

69 Marxen, GA 1980, 365, 373 (zur Unschuldsvermutung als ein „übergreifendes, für die neuzeitliche Form gesellschaftlichen Zusammenlebens konstitutives Rechtsprinzip“); ders. Straftatsystem (Fn. 10), S. 345; Paeffgen, Vorüberlegungen (Fn. 38), S. 42 ff.; Köster, Die Rechtsvermutung der Unschuld, Diss. Bonn 1979, S. 144 ff., 173 ff.; Lindner, AöR 133 (2008), S. 235 (247 ff., 257 ff.); L. Schulz, Normiertes Misstrauen (Fn. 43), S. 486 m.w.N.

70 Vgl.: BVerfGE 82, 106, 122, 124, Abw. M. Mahrenholz („Die Unschuldsvermutung verbietet jede Zweideutigkeit ..."); Frommel, Präventionsmodelle in der deutschen Strafzweck-Diskussion. Beziehungen zwischen Rechtsphilosophie, Dogmatik, Rechtspolitik und Erfahrungswissenschaften, 1987, Habil. Schrift München 1986, S. 41, 192 f. (mit einem historischen Rückblick auf die "Verwendung kriminalstatistischer Daten, die zufällig, bisweilen demagogisch, fast immer unkritisch wirkten.“); dies., in: Frommel / Löhr u.a. (Hrsg.), Neue Wege in der Kriminalpolitik, 2001, S. 4 ff., 7 ff.; Paeffgen, JR 1999, 89, 96 (zum Anspruch der Juristen, Sprache sachgerecht zu verwenden); ders., Amelung-FS (2009), S. 81, 122 (zum „Feindstrafrecht“ und zur zentralen Aufgabe von Juristen, grundsätzlich auch des Gesetzgebers, für massiv grundrechtsinvasive Befugnisse eine Grenze festzulegen).

71 Vgl.: BVerfGE 82, 106, 117 („Unabhängig davon sollten die Gerichte im Hinblick auf den verfassungsrechtlichen Rang der Unschuldsvermutung darauf Bedacht nehmen, nur solche Formulierungen zu verwenden, die von vornherein jeden Anschein einer unzulässigen Schuldzuweisung vermeiden ...“); BVerfG, NJW 2009, 350 („Die bis zur rechtskräftigen Verurteilung zu Gunsten des Angeklagten sprechende Un- 
schuldsvermutung gebietet eine zurückhaltende, mindestens aber eine ausgewogene Berichterstattung durch die Medien.“). Kühl, NJW 1984, 1264 (1267), hebt unter Berufung auf zwei EGMR-Entscheidungen hervor, dass sogar Begründungen von Einstellungsentscheidungen oder damit verbundenen Kostenentscheidungen, die „den Eindruck erwecken“ (Fall Adolf, EuGRZ 1982, 297, 302 Ziff. 38) bzw. „den Gedanken aufkommen " lassen (Fall Minelli, EuGRZ 1983, 475, 479 Ziff. 37), der Beschuldigte sei vom Gericht für schuldig betrachtet worden, die Unschuldsvermutung gemäß Art. 6
Abs. 2 EMRK verletzen. Siehe auch: Salditt, E. Müller-FS (2008) S. 611 (615, 620 f.: „Schon heute leidet der von Gerichten und Staatsanwälten verwendete lakonische Formularwortlaut vorläufiger Einstellungen (nach $\mathbb{} 153$ a StPO) daran, dass schlicht, inhaltslos und ohne Verwendung konjunktivistischer Sprachform behauptet wird, der Beschuldigte sei der ihm vorgeworfenen Straftat hinreichend verdächtig. (...) Nach der Einstellung gegen Auflagen bleibt der vormals Beschuldigte, wenn wir ihn schon nicht für schuldig halten dürfen, noch erwiesen verdächtig. Das klingt nach ungefähr unschul- dig oder fast schon nach ungefähr schuldig. (...) Unter solchen Einflüssen atrophiert die Unschuldsvermutung.“); Haeusermann, Der Verband als Straftäter und Strafprozeßsubjekt, Diss. Freiburg im Breisgau 2003, S. 226 f., 260, 264 ff., 271 (Fn. 227); Paeffgen, GA 2009, 450 (454 Fn 22: zur Unschuldsvermutung und „zu der Gedankenlosigkeit, mit der der Gesetzgeber mit dem Begriff, Täter' im Strafprozessrecht um sich wirft, noch bevor ein rechtskräftiges Urteil vorliegt.").

\section{Christine Morgenstern zu}

Norbert Kazele. Untersuchungshaft. Ein verfassungsrechtlicher Leitfaden für die Praxis. ${ }^{1}$

Wegen der überragenden Bedeutung des Freiheitsgrundrechts aus Art. 2 II GG gibt es Bundesverfassungsgerichtsentscheidungen zu den meisten Aspekten der Untersuchungshaft. Der Richter am Oberlandesbericht Norbert Kazele legt auf 130 sehr gehaltvollen Seiten ein Kompendium dieser Rechtsprechung vor, das vor allem „eine Sensibilität für die verfassungsrechtlichen Gewährleistungen zu Gunsten des einzelnen auch bei etablierten Rechtsinstituten wie der Untersuchungshaft“ (S. 5) (wieder)herstellen soll. Der kritische Blick auf die Praxis ist dabei vor allem von der Beobachtung geprägt, dass sich trotz des „drohenden Eintritts irreparabler Schäden“ durch den leichtfertigen Umgang mit Untersuchungshaft häufig genug routinemäßige Standardformulierungen bei der Anordnung oder Aufrechterhaltung finden, die eben diese gebotene Sensibilität vermissen lassen.

Der Band gliedert sich in sieben Teile: Nach den knappen einleitenden Bemerkungen zur Bedeutung des Freiheitsgrundrechts und der Unschuldsvermutung im Spannungsverhältnis zum Strafverfolgungsinteresse des Staates erörtert der Verfasser in einem kürzeren Kapitel den Einfluss der Rechtsprechung des Europäischen Gerichtshofs für Menschenrechte. In den folgenden Teilen analysiert er ausgewählte verfassungsrechtliche Fragen im Zusammenhang mit der Untersuchungshaft, insbesondere die gebotene restriktive Interpretation der Haftgründe. Sodann widmet er sich den Einwirkungen des Grundsatzes der Verhältnismäßigkeit in einem eigenen Kapitel, in diesem Zusammenhang wird vor allem das Beschleunigungsgebot thematisiert. Daran anschließend werden die Haftprüfung durch das Oberlandesgericht und formelle Gewährleistungen vor allem durch Art. 104 GG erörtert. Im letzten Teil nimmt Kazele anhand der verfassungsgerichtlichen Rechtsprechung zur Frage nach einer absoluten Höchstdauer der Untersuchungshaft Stellung.

Zunächst dürfte die Zusammenstellung der relevanten Entscheidungen des Europäischen Gerichtshofs für Menschenrechte für den Blick über den Tellerrand sehr hilfreich sein, etwa wenn darauf hingewiesen wird, dass sowohl Gerichte als auch Behörden die Judikate des EuGMR zur Kenntnis zu nehmen und umzusetzen haben (Görgülü-Entscheidung des BVerfG von 2004) und das Bundesverfassungsgericht „mittelbar im Dienst der Durchsetzung des Völkerrechts steht" (S. 34). An dieser Stelle vermisst man allerdings einen deutlicheren Hinweis darauf, dass die Rechtsprechung der beiden Gerichte, gerade in Bezug auf Fragen der Untersuchungshaft, durchaus nicht immer deckungsgleich ist: Der EuGMR geht in der Frage des Akteneinsichtsrecht nach $\$ 147$ II StPO in den Fällen Lietzow, Schöps und Garcia Alva über das vom BVerfG Gesagte deutlich hinaus, wenn die Beschränkung dieses Rechts gleichzeitig eine wirksame Bekämpfung des Haftbefehls erschwert. Eine Versagung bzw. allzu große Beschränkung des Einsichtsrechtes verstößt nach Auffassung des Gerichtshofes bei in U-Haft befindlichen Beschuldigten gegen Art. 5 (4) EMRK und den Grundsatz der Waffengleichheit (Art. 6 EMRK). Auch in Bezug auf die Berücksichtigung des Beschleunigungsgrundsatzes divergieren die Interpretationen der höchsten deutschen Gerichte und des EuGMR. ${ }^{3}$ Da ein - wenngleich seltenes - Auseinanderfallen der Rechtsprechung der Gerichte in grundrechtssensiblen Fragen hochproblematisch ist, wären Hinweise auf weiterführende Literatur wie etwa die Monographien von Esser 2002 und von Trechsel 2006 hilfreich gewesen. ${ }^{4}$

Im „nationalen Teil“ wird hingegen schnell deutlich, dass der Autor sich engagiert zu den strengen Anforderungen an Anordnung und Fortdauer der Untersuchungshaft, die das Bundesverfassungsgericht im Laufe der Jahre entwickelt hat, bekennt und dass er eine laxe Praxis in dieser Hinsicht ebenso engagiert aufs Korn nimmt. Im Rahmen dieser Besprechung sollen exemplarisch einige seiner Darlegungen zu den Haftgründen, zur Rechtfolgenerwartung in Bezug zur Verhältnismäßigkeit der Untersuchungshaft sowie zum Beschleunigungsgebot herausgegriffen werden:

Im mit „restriktive Interpretation der Haftgründe“ (S. 38 ff.) überschriebenen Abschnitt etwa kritisiert Kazele „die verbreitete Formulierung, die Wahrscheinlichkeit einer Flucht ... müsse höher sein als deren Nichteintritt“. Sie werde „der Wertigkeit des Freiheitsgrundrechts und der Unschuldsvermutung nicht gerecht“, erforderlich sei vielmehr stets eine ausreichend konkrete Tatsachengrundlage, die eine tragfähige Prognose für die Wahrscheinlichkeit einer Flucht, Verdunkelungshandlung etc. ermögliche. Ebenso deutlich legt er dar, dass die Haftgründe der Flucht bzw. Fluchtgefahr iSd $\mathbb{} 112$ Abs. 2 Nr. 1 und 2 StPO nicht stereotyp an einen Auslandsaufenthalt oder die Ausländereigenschaft geknüpft werden dürfen, sondern genau geprüft werden muss, inwieweit etwa die Verlegung des Wohnsitzes ins Ausland (z. B. zum Zwecke der Arbeitsaufnahme) tatsächlich bedeutet, dass der Betreffende sich dem Verfahren entziehen will. Besonders wichtig erscheint hier der Hinweis, dass der Umstand, dass die Aufgaben der Strafverfolgungsbehörden durch den Auslandsbezug erschwert werden können, keinen Haftgrund begründen kann (S. 41). Schließlich ist hervorzuheben wie Kazele sich mit dem Problem der Straferwartung als Indiz für bestehende Fluchtgefahr auseinandersetzt. Er bekräftigt die (unbedingt zustimmungswürdige) Auffassung nach der eine in concreto hohe Straferwartung nicht schematisch zu einer Vernachlässigung weiterer - ggf. widerstreitender - Faktoren, die bei der Beurteilung der Fluchtgefahr heranzuziehen sind, führen darf (S. 46).

Auch die differenzierte Darlegung der „Bedeutung der Sache“ und der Rechtfolgenerwartung als Bezugsgrößen für die Verhältnismäßigkeit der Anordnung bzw. Fortdauer der Untersuchungshaft 